\title{
Bioavailability of Two Oral-Granule Formulations of a Single Dose of 4-Mg Oral Granules of Montelukast: A Randomized, Two-Period Crossover Comparison in Healthy Mexican Adult Volunteers
}

Gabriel Mendoza-Tamayo', Alejandra Rosete-Reyes ${ }^{1}$, Jessica González-Bañuelos ${ }^{2}$, Ericka López-Bojórquez², Victoria Burke-Fraga² and Mario González-de la Parra*

${ }^{1}$ CIF-BIOTEC, Médica Sur, S.A.B. de C.V., Mexico City, Mexico City, Mexico

${ }^{2}$ Biokinetics, S. A. de C. V., Mexico City, Mexico

\begin{abstract}
Montelukast is a leukotriene receptor antagonist. In Mexico, the oral granules are indicated for children for the treatment of asthma and allergic rhinitis. The aim of this study was to compare the bioavailability and to determine the bioequivalence of a test formulation (oral granules) containing $4 \mathrm{mg}$ of montelukast, with its corresponding reference drug formulation, and to generate data regarding the oral bioavailability of this drug in the Mexican population. This single-dose, randomized-sequence, single-blind, two-period crossover study under fasting conditions was conducted on a total of 26 healthy Mexican adult subjects of both genders, with a seven-day washout period. Study formulations were administered after a 10-hour overnight fast. For pharmacokinetic analysis, blood samples were drawn at 0 (baseline), $0.25,0.5,1,1.5,2,2.5,3,3.5,4,5,6,8,10,12$ and 24 hours after administration. Plasma concentrations of montelukast were determined using HPLC coupled to a mass spectrometry (MS/MS) detector. The test and reference formulations were considered bioequivalent if the $90 \% \mathrm{Cls}$ for the geometric mean test/reference ratios were within a predetermined range of $80 \%$ to $125 \%$. The $90 \% \mathrm{Cls}$ for montelukast $\mathrm{C}_{\max }, \mathrm{AUC}_{0-\mathrm{t}}$ and $\mathrm{AUC}_{0-\infty}$ were $97.57 \%$ to $109.35 \%, 101.81 \%$ to $108.92 \%$, and $101.55 \%$ to $109.96 \%$, respectively. In this study a single dose of the test formulation met the regulatory requirements for assuming bioequivalence, based on the rate and extent of absorption.
\end{abstract}

Keywords: Montelukast; Bioequivalence; Bioavailability; Oralgranules

\section{Introduction}

Montelukast is a leukotriene receptor antagonist [1,2]. In Mexico, the oral granules are indicated for children for the treatment of asthma and allergic rhinitis [3].

The $4 \mathrm{mg}$ oral granules dosage form has been shown to provide systemic exposure similar to that of the $10 \mathrm{mg}$ tablet in adults [4].

Knoor et al. [5] have demonstrated that the montelukast $4 \mathrm{mg}$ oral granules formulation is bioequivalent to the $4 \mathrm{mg}$ chewable tablet formulation when administered in the fasted state. In addition, they evaluated effect of food on the pharmacokinetics of the oral granules formulation in two studies. In the first study, they found that the administration of a single $4 \mathrm{mg}$ dose of the oral granules formulation with applesauce, a food commonly consumed by young children, had no effect on overall drug exposure, as indicated by the nearly identical $\mathrm{AUC}_{0-\infty}$ values observed when the oral granules formulation was administered with and without applesauce.

In the second study, they evaluated the effect of the oral granules formulation after consumption of a high-fat breakfast; the findings of this study were that the consumption of a high-fat breakfast had no evident effect on the extent of absorption, as indicated by the similarity between $\mathrm{AUC}_{0-\infty}$ values after administration of the oral granules formulation with and without food. However, the $\mathrm{C}_{\max }$, was statistically significantly lower (36\%) and the $\mathrm{T}_{\max }$ delayed (from 2 to 6 hours) after administration with a high-fat breakfast. It is important to point out that the authors acknowledged that it is unlikely that a meal with such a high-fat and calorie content would be consumed by 6 months to 2-year-old patients, and that the delay in absorption should not be regarded as clinically important for a chronically administered drug like montelukast, whose pharmacological effects are more dependent on extent of systemic exposure (i.e., AUC) rather than on maximum peak plasma concentrations [5].

The sponsor of this study (Laboratorios Liomont, S. A. de C. V.) was interested in obtaining the marketing authorization for montelukast 4 $\mathrm{mg}$, as a granule formulation (test formulation) in Mexico (Everest ${ }^{\circ}$, Laboratorios Liomont, S. A. de C. V., Mexico City, Mexico).

Therefore, the aim of this study was to compare the bioavailability and to determine the bioequivalence of a test formulation containing 4 mg of montelukast, with its corresponding reference drug formulation (Singulair granules. Merck Sharp \& Dohme de Mexico, S. A. de C. V.).

A search of PubMed, MEDLINE and Google data bases for literature published up to May of 2014, using the combination terms montelukast, bioequivalence, bioavailability, pharmacokinetics, oral-granules, Mexico, Mexican and population, did not identify any published data concerning the bioavailability of oral montelukast in the Mexican population.

\section{Materials and Methods}

*Corresponding author: Mario González-de la Parra, Pvda. Jesus del Monte No. 77, Col. Cuajimalpa, 05000 Mexico D.F., Mexico, E-mail: mdelaparra@biokinetics.com.mx Received August 06, 2015; Accepted October 02, 2015; Published October 10 2015

Citation: Mendoza-Tamayo G, Rosete-Reyes A, González-Bañuelos J, LópezBojórquez E, Burke-Fraga V, González-de la Parra M (2015) Bioavailability of Two Oral-Granule Formulations of a Single Dose of 4-Mg Oral Granules of Montelukast: A Randomized, Two-Period Crossover Comparison in Healthy Mexican Adult Volunteers. J Bioequiv Availab 7: 274-278. doi:10.4172/jbb.1000253

Copyright: ( $) 2015$ Mendoza-Tamayo G, et al. This is an open-access article distributed under the terms of the Creative Commons Attribution License, which permits unrestricted use, distribution, and reproduction in any medium, provided the original author and source are credited. 
The study protocol (P456S026V004) and the informed-consent form were reviewed and approved by an independent ethics and research committee (Comité de Ética e Investigación para Estudios en Humanos, Mexico City, Mexico) on November 26, 2012, and by COFEPRIS (Federal Commission for Protection against Sanitary Risks, for its Spanish acronym) on January 31, 2013. The study was conducted in accordance with the ethical principles of the Declaration of Helsinki and its amendments and the International Conference on Harmonisation for Good Clinical Practice Guideline.

The principal investigator informed the subjects of all procedures, duration of the study, anticipated risks and discomfort it could entail, and an individual written informed consent was obtained prior to the initiation of the study. The study was conducted from April to June of 2013.

\section{Inclusion/exclusion criteria}

Healthy Mexican adults aged 18 to 55 years and of either gender were eligible for inclusion in the study. Subjects were recruited from the volunteer's database at the Center of Pharmacological and Biotechnology Research (clinical unit) in Medica Sur Hospital, Mexico City, Mexico.

Each potential subject had a clinical health evaluation. Classification of subjects as healthy was based on unremarkable findings obtained in a personal interview; a complete physical examination (blood pressure [BP], heart rate, weight, height, temperature and respiratory rate); and diagnostic testing that included a 12-lead ECG, chest radiography, and laboratory blood chemistry test, serological tests for hepatitis B and $\mathrm{C}$ and HIV antibodies, urinalysis, alcohol test, drug-abuse tests and a pregnancy test in women. Other exclusion criteria were alcohol consumption (abstinence during the entire duration of the study); and tobacco consumption (abstinence for at least 48 hours prior to the initiation of the study and during its entire duration).

Systolic and diastolic BP was measured with a sphygmomanometer (Tycos; Welch Allyn, Skaneateles Falls, NY). The BP cuff was applied to the right arm and the reading was taken with the subject in a seated position. Candidates were excluded if laboratory values were significantly out of the reference range and/or if all tests had not been completed. Laboratory testing was performed at Medica Sur Hospital, which has been certified by the Mexican government and the College of American Pathologists. The scope of the certifications included the tests relevant to this study. Before the enrollment of the participants, the laboratory data were reviewed by investigators at the clinical unit.

\section{Study design and drug administration}

A single-dose randomized-sequence, single-blind, two-period crossover design under fasting conditions was used. The subjects were admitted to the clinical site on the day before the drug administration, and were randomly assigned by a pharmacist in the presence of quality assurance personnel at the clinical unit in a 1:1 ratio, using a computergenerated table of random numbers to one of the two sequences (test formulation containing montelukast sodium equivalent to $4 \mathrm{mg}$ of montelukast (lot 239D0017; expiration date January 2015) followed by the reference formulation containing montelukast sodium equivalent to $4 \mathrm{mg}$ of montelukast (lot H013658; expiration date April 2014), or vice versa.

To ensure reliable baseline plasma measurements, participants underwent a 10 hour overnight fast. Based on the reported mean plasma half-life of montelukast, which ranges from 2.7 to 5.5 hours
[3], a seven-day washout period was considered appropriate because it exceeds the seven half-lives required by COFEPRIS [6].

Blood samples were drawn for baseline plasma determinations in the following way. An 18-GA x 1.16 in $(1.3 \times 30 \mathrm{~mm})$ indwelling angiocatheter (BD-InSyte, Becton Dickinson Ind. Cir. Ltda, Minas Gerais, Brazil) was inserted in a suitable forearm vein and a 7.5 $\mathrm{mL}$ blood sample was drawn into a heparin-treated vacuum tube (S-Monovette, Sarstedt AG \& Co., Nümbrecht, Germany).

Subjects were administered a sachet containing $4 \mathrm{mg}$ oral granules of the test or the reference formulation to be placed in the oral cavity and ingested with the aid of $250 \mathrm{ml}$ of water. Additional blood samples were drawn at $0.25,0.5,1,1.5,2,2.5,3,3.5,4,5,6,8,10,12$ and 24 hours after administration.

During hospitalization, the subjects were under medical surveillance, and during the washout period participants maintained contact with the investigators to report any adverse events (AEs).

Plasma was obtained by centrifugation ( $3000 \mathrm{rpm}$ for 15 minutes at room temperature) and stored at $-70^{\circ} \mathrm{C} \pm 10^{\circ} \mathrm{C}$ (until it was transported to the analytical unit where it was stored at $-75^{\circ} \mathrm{C} \pm 5^{\circ} \mathrm{C}$ until it was analyzed). After a seven-day washout period, participants returned to the clinical unit, where the alternative formulation was administered as in the first treatment period.

Subjects were asked to refrain from water and food intake for three hours after the study drug administration. Their diet, for each treatment period, consisted of three standardized meals $(2113 \mathrm{kcal} / \mathrm{d})$ at 3.25, 6.5 and 12.75 hours after the study drug administration.

\section{Determination of montelukast plasma concentrations}

Chemicals: Montelukast sodium (lot: 10-202, purity 98.2\%) secondary standard was obtained from Sintenovo (Estado de Mexico, Mex.) and loratadine reference standard from the USP (Rockville, MD). All solvents (including water) were HPLC-mass spectrometric grade (Avantor Performance Materials, Inc., Phillipsburg, NJ) and all reagents were analytical grade (Mallinckrodt Baker, Inc., Phillipsburg, NJ).

Method and sample preparation: Montelukast sodium plasma levels were determined as montelukast (the free acid form of the montelukast sodium salt) by using a HPLC method coupled with mass spectrometry (MS/MS) developed and validated by personnel of Biokinetics in Mexico City, Mexico. The method included the following: $250 \mu \mathrm{L}$ of plasma, $10 \mu \mathrm{l}$ of internal standard (loratadine, $0.05 \mu \mathrm{g} / \mathrm{mL}$ ) and $750 \mu \mathrm{L}$ of acetonitrile. These components were vortexed in a 2.0 $\mathrm{mL}$ conical tube (Sarstedt AG \& Co.) for one minute. The tube was centrifuged at $8000 \mathrm{rpm}$ for five minutes at room temperature $\left(25^{\circ} \mathrm{C}\right)$. The supernatant was separated and injected (volume of injection $=3$ $\mu \mathrm{L}$ ) into the chromatographic system (HPLC, Agilent Technologies, model 1200, Palo Alto, California).

Chromatographic Conditions: Montelukast concentrations were determined with a $50 \times 4.6$ - $\mathrm{mm}$ internal-diameter column of $1.8 \mu \mathrm{m}$ particle size (Zorbax Eclipse XDB-C8, Agilent Technologies) and eluted with a mobile phase consisting of a mixture $(30: 70 \mathrm{v} / \mathrm{v})$ of an aqueous buffer solution (ammonium formate, $10 \mathrm{mM}$; $\mathrm{pH} 6.0$ \pm 0.1 ) and acetonitrile. The column temperature was $25^{\circ} \mathrm{C}$. Flow rate was maintained at $0.6 \mathrm{~mL} /$ minute and the montelukast and loratadine were detected by a triple-quadrupole mass spectrometer (Agilent Technologies, model G6410B). The spectrometric (MS/MS) analysis was performed by monitoring the transitions $586.2 \rightarrow 422.3 .1$ $\mathrm{m} / \mathrm{z}$ for montelukast and $383.3 \rightarrow 337.2 \mathrm{~m} / \mathrm{z}$ for loratadine (IS). The 
spectrometric conditions were: positive-ionization mode, fragmenter energy (100 and $120 \mathrm{~V}$ for montelukast and IS, respectively), collision energy $(25 \mathrm{~V})$, drying gas (nitrogen at $\left.350^{\circ} \mathrm{C}\right)$, gas flow $(10 \mathrm{~L} /$ minute) and pressure (45 psi). Typical retention times for montelukast and the internal standard were 3.0 and 2.1 minutes, respectively. Montelukast peak areas were used for its quantitation.

Method validation: The analytical method was validated according to Mexican and international guidelines [6,7].

The selectivity of the method was tested by the analysis of blank human plasma samples from six different subjects, blank human (hemolyzed and lipemic) plasma samples, as well as anticoagulants (heparin), xanthines (caffeine and theobromine), and other drug substances commonly used as analgesics (acetylsalicylic acid, ibuprofen, diclofenac, paracetamol and naproxen). No interferences were observed in the resulting chromatograms.

The range of the method was 0.003 to $0.150 \mu \mathrm{g} / \mathrm{mL}$, with lower limits of quantification (LLOQ) and of detection (LLOD) of 0.003 and $0.001 \mu \mathrm{g} / \mathrm{mL}$, respectively. The method was found to be linear within this range of concentrations with a coefficient of determination of 0.99 . The intra-assay \%CV and accuracy (relative error) for montelukast were $1.09 \%$ to $3.37 \%$ and $-9.22 \%$ to $-6.73 \%$, respectively, while the inter-assay $\% \mathrm{CV}$ and accuracy were $2.89 \%$ to $5.03 \%$ and $-8.47 \%$ to $-8.17 \%$. The absolute recovery was above $87 \%$.

Montelukast in plasma was found to be stable after 24 hours at room temperature $\left(25^{\circ} \mathrm{C}\right)$, after three freeze-thaw cycles and after 16 weeks at $-75 \pm 5^{\circ} \mathrm{C}$

Quality control (QC) samples were included in every analytical run to verify its performance. These QC samples were prepared at three different concentration levels (designated as low $(0.01 \mu \mathrm{g} / \mathrm{mL})$, medium $(0.06 \mu \mathrm{g} / \mathrm{mL})$ and high $(0.115 \mu \mathrm{g} / \mathrm{mL}))$ of montelukast independent of the calibration curve. This method was considered suitable by the investigators for the bioequivalence study of montelukast.

Tolerability: Tolerability was determined using clinical assessment, monitoring of vital signs at baseline, 2 and 6 hours after the drug administration during hospitalization, and at the end of the clinical stage of the study.

The subjects were interviewed (using open-ended questions) by the investigators during the trial conduct and at the end of the clinical stage of the study, concerning the occurrence AEs. Subjects were asked to spontaneously report any $\mathrm{AE}$ to the investigators at any time during the study, including the washout period. Data for all AEs were recorded on a case-report form.

AEs that were life-threatening, led to death, hospitalization, disability, and/or medical intervention to prevent permanent impairment or damage, were considered serious.

Pharmacokinetic and statistical analyses: Sample size calculation [8] was based on the within-subject variability of montelukast $\mathrm{C}_{\max }$ with a \% CV of $21.2 \%$ [5]. This calculation was performed considering the following values: $1-\beta=0.8, \alpha=0.05, \% \mathrm{CV}=21.2$, and an equivalence range of $80 \%$ to $125 \%$, yielded with a sample size of 22 subjects. In this study, a sample size of 26 subjects was used because, at the time the study was conducted, a minimum sample size of 24 subjects was required for bioequivalence studies by COFEPRIS.

Individual plasma concentration-time curves were constructed; $\mathrm{C}_{\max }$ and $\mathrm{T}_{\max }$ were directly obtained from these curves, the area under the plasma concentration-time curve from time baseline to the last measurable concentration $\left(\mathrm{AUC}_{0-\mathrm{t}}\right)$ was calculated by a noncompartmental method using the trapezoidal rule. From the terminal log-decay phase, the elimination rate constant (ke) was estimated using linear regression, and the $t^{1 / 2}$ was estimated using the following equation [8]:

$\mathrm{t}^{1 / 2}=\ln 2 / \mathrm{ke}$, where $\ln$ was defined as the natural logarithm. Extrapolation of AUC from baseline to infinity $\left(\mathrm{AUC}_{0-\infty}\right)$ was calculated as follows:

$$
\mathrm{AUC}_{0-\infty}=\mathrm{AUC}_{0-\mathrm{t}}+\mathrm{Ct} / \mathrm{ke} \text {, }
$$

Where Ct was the last measurable plasma concentration.

To assess the bioequivalence between the test and reference formulations, $\mathrm{C}_{\max }, \mathrm{AUC}_{0-\mathrm{t}}$ and $\mathrm{AUC}_{0-\infty}$ were considered as the primary variables. ANOVA for a 2 × 2 crossover design, using log-transformed data for these parameters, was carried out at the $5 \%$ significance level $(\alpha=0.05)$.

The $90 \%$ CIs of the geometric mean ratios (test/reference) of $\mathrm{C}_{\text {max }^{2}}$ $\mathrm{AUC}_{0-\mathrm{t}}$ and $\mathrm{AUC}_{0-\infty}$ were calculated using log-transformed data. The test and the reference formulations were considered bioequivalent if the $90 \%$ CIs of these parameters fell within a predetermined range of $80 \%$ to $125 \%$. All pharmacokinetic and statistical analyses were performed using WinNonlin Version 5 (Pharsight, Mountain View, California).

\section{Results}

Table 1 show the demographic characteristics for a total of 26 subjects, who were enrolled and completed the clinical stage of the study.

\section{Pharmacokinetic parameters}

Mean plasma concentration-time curves of the two formulations are shown in Figure 1. This figure suggests comparable mean plasma concentration-time curves.

Table 2 shows the pharmacokinetic parameters $\left(C_{\max }, T_{\max }, t_{1 / 2}\right.$, $\mathrm{AUC}_{0-\mathrm{t}}$ and $\left.\mathrm{AUC}_{0-\infty}\right)$ for both formulations.

No significant period or sequence effects were detected based on the ANOVA of $\mathrm{C}_{\max }, \mathrm{AUC}_{0-\mathrm{t}}$ and $\mathrm{AUC}_{0-\infty}$ (data not provided).

Table 3 shows the bioequivalence statistics using the logtransformed data of $\mathrm{C}_{\max }, \mathrm{AUC}_{0-\mathrm{t}}$ and $\mathrm{AUC}_{0-\infty}$ : geometric mean ratios (test $/$ reference), $90 \% \mathrm{CI}$, and the intra-subject $\% \mathrm{CV}$.

The $90 \%$ CIs for montelukast $\mathrm{C}_{\max }, \mathrm{AUC}_{0-\mathrm{t}}$ and $\mathrm{AUC}_{0-\infty}$ were $97.57 \%$ to $109.35 \%, 101.81 \%$ to $108.92 \%$, and $101.55 \%$ to $109.96 \%$, respectively. The $90 \%$ CIs of the geometric mean ratios of the three parameters fell within the predetermined range of $80 \%$ to $125 \%$.

These results indicate that the bioequivalence criteria were met.

\section{Tolerability}

Four of the 26 subjects reported a total of 5 AEs. All of them were reported after the administration of the reference formulation: two

\begin{tabular}{|l|c|}
\hline Characteristic & Values \\
\hline Total No. of subjects (female/male) & $26(11 / 15)$ \\
\hline Age, mean (SD), range, years & $31(8), 18-50$ \\
\hline Weight, mean (SD), range, $\mathrm{kg}$ & $64.66(7.04), 53.20-75.70$ \\
\hline Height, mean (SD), range, $\mathrm{m}$ & $1.66(0.08), 1.51-1.80$ \\
\hline BMI, mean (SD), range, $\mathrm{kg} / \mathrm{m}^{2}$ & $23.42(1.33), 21.00-26.04$ \\
\hline
\end{tabular}

$\mathrm{BMI}=$ Body mass index

Table 1: Demographic characteristics of subjects. 


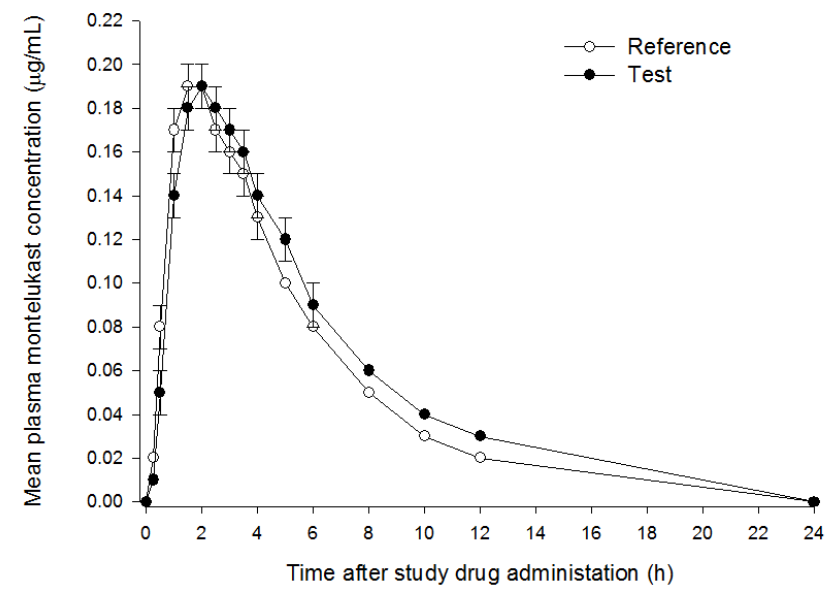

Figure 1: Mean $( \pm S E)$ plasma concentration-time curves after a single-dose administration of a test (trademark: Everest ${ }^{\circledR}$, Laboratorios Liomont, S. A. de C. V.) and a reference (trademark: Singulair ${ }^{\circledR}$, Merck Sharp \& Dohme de Mexico, S. A. de C. V.) oral 4-mg granule formulation, containing montelukast in healthy Mexican adult subjects $(n=26)$.

\begin{tabular}{|c|c|c|}
\hline Parameter & Test $^{*}$ & Reference $^{\dagger}$ \\
\hline $\mathrm{C}_{\text {max }}, \mu \mathrm{g} / \mathrm{ml}$ & $0.21(0.04)$ & $0.21(0.05)$ \\
\hline $\mathrm{AUC}_{0-\mathrm{t}}, \mu \mathrm{g} \bullet \mathrm{h} / \mathrm{ml}$ & $1.10(0.23)$ & $1.05(0.23)$ \\
\hline $\mathrm{AUC}_{0-\infty}, \mu \mathrm{g} \bullet \mathrm{h} / \mathrm{ml}$ & $1.23(0.28)$ & $1.17(0.29)$ \\
\hline $\mathrm{T}_{\max }, \mathrm{h}$ & $2.17(0.86)$ & $1.67(0.68)$ \\
\hline $\mathrm{t}_{1 / 2}, \mathrm{~h}$ & $3.24(0.48)$ & $3.32(0.94)$ \\
\hline
\end{tabular}

$\mathrm{C}_{\text {maximum }}=$ Masma drug concentration

AUC =AUC from time 0 (baseline) to the last measurable concentration $A \cup C_{0-\infty}^{0-t}=A \cup C$ from baseline extrapolated to infinity

${ }^{*}$ Trademark: Everest ${ }^{\circledR}$ (Laboratorios Liomont, S. A. de C. V., Mexico City, Mexico).

†Trademark: Singulair ${ }^{\circledR}$ (Merck Sharp \& Dohme de Mexico, S. A. de C. V., Mexico City, Mexico).

Table 2: Pharmacokinetic parameters of a test and a reference formulation of oral montelukast $4 \mathrm{mg}$ after a single-dose administration in healthy Mexican adult subjects $(n=26)$. Values are mean $(S D)$.

\begin{tabular}{|c|c|c|c|}
\hline Parameter & $\begin{array}{c}\text { Geometric Mean } \\
\text { Ratio (\%) }\end{array}$ & $\mathbf{9 0 \%} \mathbf{C l}$ & $\begin{array}{c}\text { Intra-subject } \\
\text { \% CV }\end{array}$ \\
\hline $\mathrm{C}_{\max }$ & 103.29 & $97.57 ; 109.35$ & 12.05 \\
\hline $\mathrm{AUC}_{0-\mathrm{t}^{\prime}}$ & 105.31 & $101.81 ; 108.92$ & 7.12 \\
\hline $\mathrm{AUC}_{0-\infty}$ & 105.67 & $101.55 ; 109.96$ & 8.40 \\
\hline
\end{tabular}

$\mathrm{C}_{\text {. }}=$ Maximum plasma drug concentration

AUC $=$ AUC from time 0 (baseline) to the last measurable concentration

$\mathrm{AUC}_{0-\infty}=\mathrm{AUC}$ from baseline extrapolated to infinity

Table 3: Geometric mean ratios, $90 \% \mathrm{Cls}$ and the intra-subject $\% \mathrm{CV}$ of the pharmacokinetic parameters determined for montelukast after a single-dose administration of $4 \mathrm{mg}$ in healthy Mexican adult subjects.

headaches, one skin rash, one conjunctivitis, and one acute stress.

None of the AEs was considered serious. Instead, all of them were regarded as mild, and all of the AEs spontaneously resolved under medical surveillance during the clinical stage.

\section{Discussion}

All of the $90 \%$ CIs of the geometric mean ratios of the pharmacokinetic parameters $\left(\mathrm{C}_{\max }, \mathrm{AUC}_{0-\mathrm{t}}\right.$ and $\left.\mathrm{AUC}_{0-\infty}\right)$ were found to be within the predetermined range of bioequivalence (80\%-125\%). These results indicate that the bioequivalence criteria were met.

Both formulations were well tolerated, in view of that fact that none of the reported AEs by four subjects was considered serious and all had been previously reported.

As with any clinical trial, and in particular with most bioavailability studies, the current study had some limitations that should be considered. First, this was a single-blind study, so it might not objectively address the effectiveness and safety profiles of the formulations tested.

The data were obtained from healthy adult subjects, in accordance with regulatory requirements (COFEPRIS), within a specific age range, who were administered a single dose of the formulation. The PK parameters of montelukast might differ in target populations (e.g. children). For example, differences in absorption, metabolism and excretion of montelukast might exist in pediatric patients, with respect to adult subjects. The pharmacokinetics of montelukast might also differ among patients with different genotypes [9]. Thus, the results of this study might not be generalizable to a target population.

In addition, the effect of food was not evaluated because it has been reported that the extent of the absorption of montelukast (as indicated by AUC values) is not affected by food intake, especially when considering the typical diet of young children [5].

Further studies are needed to compare the test formulation with the reference formulation in Mexican patient groups. The results of this study might serve as a reference for future controlled studies of montelukast in a Hispanic population.

\section{Conclusions}

In this study of healthy, fasting, Mexican adult subjects, who received a single dose of either the test or reference formulation, it was concluded that the test formulation of 4-mg oral granules of montelukast met the Mexican regulatory requirements to assume bioequivalence, based on the rate and extent of absorption. Both formulations were well tolerated.

\section{Acknowledgements}

This research and its publication were supported by Laboratorios Liomont S.A. de C.V., Mexico City, Mexico. The authors have indicated that they have no conflicts of interest regarding the content of the article.

\section{References}

1. Benninger MS, Waters $\mathrm{H}$ (2009) Montelukast: Pharmacology, Safety, Tolerability and Efficacy, Clinical Medicine. Therapeutics 1: 1253-1261.

2. Jones TR, Labelle M, Belley M, Champion E, Charette L, et al. (1995) Pharmacology of montelukast sodium (Singulair ${ }^{\mathrm{TM}}$ ), a potent and selective leukotriene D4 receptor antagonist. Canadian Journal of Physiology and Pharmacology 73: 191-201.

3. Merck Sharp \& Dohme de Mexico, S. A. de C. V (2013) Prescribing information (in Spanish) Singulair ${ }^{\circledR}$, Diccionario de Especialidades Farmaceuticas $\left(\mathrm{PLM}^{\circledR}\right)$ $59^{\text {th }}($ Edn), Mexico City, Mexico: 3091-3096.

4. Migoya E, Kearns GL, Hartford A, Zhao J, van Adelsberg J, et al. (2004) Pharmacokinetics of montelukast in asthmatic patients 6 to 24 months old. Clin Pharmacol 44: 487-494.

5. Knorr B, Hartford A, Li XS, Yang AY, Noonan G, et al. (2010) Bioequivalence of the 4-mg Oral Granules and Chewable Tablet Formulations of Montelukast. See comment in PubMed Commons below Arch Drug Inf 3: 37-43.

6. COFEPRIS, Federal Commission for the Protection against Sanitary Risks (1999) Official Mexican Standard NOM 177-SSA1-1998, Tests and procedures to prove that a medication is interchangeable [in Spanish], General Directorate of Standards, Mexico City, Mexico.

7. Gao L, Li J, Kasserra C, Song Q, Arjomand A, et al. (2011) Precision and accuracy in the quantitative analysis of biological samples by accelerator mass spectrometry: application in microdose absolute bioavailability studies. Anal Chem 83: 5607-5616.

8. Chow SC, Liu JP (2000) Design and Analysis of Bioavailability and 
Citation: Mendoza-Tamayo G, Rosete-Reyes A, González-Bañuelos J, López-Bojórquez E, Burke-Fraga V, González-de la Parra M (2015) Bioavailability of Two Oral-Granule Formulations of a Single Dose of 4-Mg Oral Granules of Montelukast: A Randomized, Two-Period Crossover Comparison in Healthy Mexican Adult Volunteers. J Bioequiv Availab 7: 274-278. doi:10.4172/jbb.1000253

Bioequivalence Studies. $2^{\text {nd }}($ Edn) New York, NY: Marcel Dekker.
9. Mougey EB, Feng H, Castro M, Irvin CG, Lima JJ (2009) Absorption of montelukast is transporter mediated: a common variant of OATP2B1 is associated with reduced plasma concentrations and poor response. Pharmacogenet Genomics 19: 129-138. 